\title{
Perdas de solo e água em um Argissolo Vermelho Amarelo, submetido a diferentes intensidades de chuva simulada ${ }^{1}$
}



Daniel F. de Carvalho ${ }^{2}$, Claudinei A. Montebeller ${ }^{3}$, Eleandro S. da Cruz ${ }^{3}$, Marcos B. Ceddia ${ }^{4}$ \& Ângela M. Q. Lana ${ }^{5}$

\author{
1 Trabalho financiado pelo $\mathrm{CNPq}$ \\ 2 DE/IT/UFRRJ. BR 465, km 7. Seropédica, RJ. CEP: 23.851-970. Bolsista CNPq. E-mail:carvalho@ufrrj.br (Foto) \\ ${ }^{3}$ Graduando, UFRRJ. Bolsista de Iniciação Científica - PIBIC/UFRRJ/CNPq \\ ${ }^{4}$ DS/IA/UFRRJ. E-mail: ceddia@ufrrj.br \\ ${ }^{5}$ Escola de Veterinária/Departamento de Zootecnia/UFMG. E-mail: lana@vet.ufmg.br
}

Protocolo $178-18 / 12 / 2001$

\begin{abstract}
Resumo: Este trabalho foi desenvolvido com o objetivo de se estimar as perdas de solo e água em um Argissolo Vermelho Amarelo, utilizando-se um simulador de chuvas pendular. Para testes com 35 min de duração, o simulador foi regulado adequadamente, a fim de aplicar chuvas com cinco diferentes valores de energia cinética $\left(138,184,229,275\right.$ e $321 \mathrm{~J} \mathrm{~m}^{-2}$ ) correspondendo às intensidades de $30,40,50,60$ e $70 \mathrm{~mm} \mathrm{~h}^{-1}$, respectivamente. Com os resultados obtidos, pôde-se ajustar equações de regressão entre as perdas de solo e de água, e o tempo de precipitação. Utilizando-se as equações ajustadas, obtiveram-se valores de perda que variaram de 2,83 a $26,82 \mathrm{~g} \mathrm{~m}^{-2}$ (solo) e de 0,00209 a $0,01370 \mathrm{~m}^{3} \mathrm{~m}^{-2}$ (água) quando a energia cinética da chuva variou de 138 para $321 \mathrm{~J} \mathrm{~m}^{-2}$, respectivamente. Comparando-se os valores simulados com os dados de campo, verificaram-se variações máximas de 3,4 e 5,7\%, para as perdas de solo e água, respectivamente.
\end{abstract}

Palavras-chave: perda de solo, erosão, simulador de chuva

\section{Soil and water losses in a Red Yellow Argissol under different intensities of simulated rainfall}

\begin{abstract}
The objective of this research was to estimate soil and water losses of a Red Yellow Argissol, using a pendulum rainfall simulator. Rainfall was simulated for $35 \mathrm{~min}$ at the intensities of $30,40,50,60$ and $70 \mathrm{~mm} \mathrm{~h}^{-1}$, which corresponded to kinetic energy values of 138, 184, 229, 275 and $321 \mathrm{~J} \mathrm{~m}^{-2}$, respectively. Cumulative soil and water losses were recorded with time at $5 \mathrm{~min}$ intervals. The results of soil and water losses were then fitted by regression equations. The fitted equations yielded soil and water losses that varied from 2.83 to $26.82 \mathrm{~g} \mathrm{~m}^{-2}$ (for soil) and from 0.00209 to $0.01370 \mathrm{~m}^{3} \mathrm{~m}^{-2}$ (for water) when the rainfall kinetic energy varied from 138 to $321 \mathrm{~J} \mathrm{~m}^{-2}$. Fitted and field measured soil and water losses showed a maximum variation of 3.4 and $5.7 \%$, respectively.
\end{abstract}

Key words: soil loss, erosion, rainfall simulator

\section{INTRODUÇÃO}

A degradação dos solos tanto afeta as terras agrícolas como as áreas com vegetação natural intensamente explorada pelo homem e pode ser considerada um dos mais importantes problemas ambientais dos nossos dias. Dentre os tipos de degradação, a erosão hídrica é considerada a que mais tem contribuído para a improdutividade dos solos, facilitada e acelerada pelo homem com suas práticas inadequadas de manejo agrícola.

O processo erosivo consiste de três eventos seqüenciais caracterizados pelo desprendimento, arraste e deposição das partículas do solo. O desprendimento é definido como a liberação de partículas dos agregados e, uma vez desprendidas elas podem permanecer próximas ao agregado ou serem transportadas (Kinnel \& Cummings, 1993; Amorim, 1999). Segundo Bahia et al. (1992) o desprendimento ou desagregação das partículas ocorre pelo efeito integrado da energia de impacto das gotas d'água e da força cisalhante do escoamento superficial constituindo, assim, o estágio inicial e mais importante do processo da erosão hídrica.

A erosão hídrica pode ocorrer na forma laminar, sulcos e voçorocas. A erosão laminar se caracteriza pela remoção de camadas delgadas da superfície do solo, apenas sendo 
perceptível após uma grande quantidade de solo ter sido removida. Amorim et al. (2001) se refere a essa forma de erosão como sendo a erosão entre sulcos, diferindo-a da erosão em sulcos, que se caracteriza pela formação de pequenas irregularidades no sentido da declividade do terreno, fazendo com que o escoamento superficial se concentre nos pontos mais baixos, atingindo volume e velocidade suficientes para formar canais mais ou menos profundos. Em complemento, caracteriza-se como voçorocas, o deslocamento de grande quantidade de solo com a formação de canais de grandes dimensões, impedindo o trânsito de máquinas e reduzindo a área de plantio (Pruski, 1996).

Tanto para as condições de chuva natural quanto para chuva simulada ou irrigação por aspersão, gotas de água de diferentes tamanhos podem alcançar diferentes velocidades de queda, fazendo com que a superfície do solo fique sujeita ao impacto dessas gotas com energia de diferentes magnitudes. O impacto das gotas, associado ao escoamento superficial, constitui o mecanismo responsável pela erosão entre sulcos ou laminar.

Para muitos solos, as partículas desprendidas no processo erosivo formam uma camada compactada (superfície de selamento) contribuindo para a redução da taxa de infiltração da água no solo (Chaves et al., 1993). Segundo Reichert et al. (1992) a textura do solo afeta sensivelmente o salpico de partículas com o impacto das gotas de chuva, o que contribui para uma redução da porosidade da camada superficial do solo. Além do impacto da gota, Morin \& van Winkel (1996) citam a dispersão físico-química das argilas do solo como causas da formação do selamento superficial e, conseqüentemente, da redução da velocidade de infiltração. Por outro lado, a manutenção de resíduos culturais sobre a superfície do solo, elevando a sua rugosidade, associada à pequena movimentação mecânica do solo, favorece um controle mais eficaz da erosão hídrica. Segundo Leite et al. (2002) os preparos conservacionistas possibilitam, de modo geral, maior retenção dos resíduos, promovendo aumento da tensão crítica de cisalhamento e, em consequiência, a resistência do solo à erosão hídrica.

Diferentes tipos de solo podem apresentar susceptibilidade diferenciada à erosão, mesmo para condições semelhantes de declividade, cobertura vegetal e práticas de manejo. Essas diferenças são devidas às propriedades do próprio solo e são denominadas erodibilidade do solo (Bertoni \& Lombardi Neto, 1985). Os mesmos autores classificam o fenômeno como sendo a vulnerabilidade do solo ou a sua susceptibilidade à erosão, que é a recíproca da sua resistência à erosão. As propriedades do solo que mais influenciam a sua erodibilidade, são aquelas que afetam a taxa de infiltração da água no solo, associada à sua resistência ao cisalhamento.

Dentro deste contexto, desenvolveu-se este trabalho com o objetivo de se estimar as perdas de solo e água em um
Argissolo submetido a chuvas com diferentes intensidades, utilizando-se um simulador de chuvas pendular.

\section{MATERIAL E MÉTODOS}

Na condução do experimento foi utilizado um simulador de chuvas pendular, pertencente ao Departamento de Engenharia da UFRRJ, que aplica chuvas em uma parcela útil de $0,80 \mathrm{~m}^{2}$. Em função da avaliação preliminar feita no equipamento com relação ao tipo de bico, pressão de serviço e velocidade de ciclo, selecionou-se o bico "Vee-Jet" 80.150, submetido à pressão de $34,0 \mathrm{kPa}$ a fim de simular uma chuva com características próximas às da chuva natural (Montebeller et al., 2001) . Trabalhando com esta combinação de bico e pressão, o equipamento foi regulado, a fim de aplicar chuvas com cinco diferentes valores de energia cinética $(138,184,229,275$ e $321 \mathrm{~J} \mathrm{~m}^{-2}$ ) as quais foram determinadas por meio de um programa computacional desenvolvido por Alves Sobrinho et al. (2001). Os diferentes valores de energia cinética foram conseguidos regulando-se o tempo de movimentação do pêndulo do simulador, os quais foram de 18,$5 ; 25,5 ; 31,6 ; 36,4 \mathrm{e}$ 42,9 ciclos $\mathrm{min}^{-1}$, correspondendo às intensidades de 30,40 ,

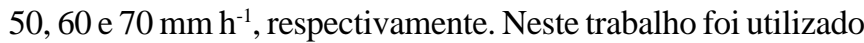
um delineamento em blocos casualizados com 5 tratamentos (chuvas com diferentes valores de energia cinética) e 4 repetições.

Os testes foram realizados em uma área experimental do Sistema de Produção Agro-Ecológico (SIPA) - EMBRAPA/ UFRRJ/PESAGRO, com uma declividade média de 8,0\%, cujo solo foi classificado como Argissolo Vermelho Amarelo. Algumas de suas características físico-químicas estão apresentadas na Tabela 1. Em função de um manejo agroecológico, esta área recebe incorporações frequientes de restos culturais sendo que, antes do início dos testes, ela foi roçada, pois estava sendo ocupada com o feijão de porco (Canavalia ensiformis L.).

Com o uso de um regador, promoveu-se a um pré-molhamento que teve, como objetivo, homogeneizar as condições de umidade do solo nas parcelas. A lâmina de água foi aplicada até se observar o início do escorrimento superficial e correspondeu, em média, a uma lâmina de $10 \mathrm{~mm}$. O teste teve início $10 \mathrm{~min}$ após o pré-molhamento. Com as regulagens apresentadas, foram simuladas chuvas com 35 min de duração, sendo coletadas sete amostras em cada parcela, em recipientes de $1 \mathrm{~L}$, em intervalos de $5 \mathrm{~min}$. Portanto, conforme metodologia apresentada por Varella (1999) os tempos de coleta corresponderam a 5, 10, 15, 20, 25, 30 e $35 \mathrm{~min}$, a partir do início da chuva. No final de cada teste, as amostras foram encaminhadas ao laboratório e ficaram em frascos vedados até que fosse feita a estimativa da quantidade de água e solo presentes nas mesmas. Este procedimento foi realizado no Laboratório de Física e Classificação de Solo do Instituto de Agronomia da UFRRJ,

Tabela 1. Algumas características físicas e químicas do solo utilizado

\begin{tabular}{|c|c|c|c|c|c|c|c|c|c|c|c|}
\hline \multirow{2}{*}{ Horiz. } & \multirow{2}{*}{$\begin{array}{l}\text { Prof. } \\
(\mathrm{cm})\end{array}$} & \multicolumn{4}{|c|}{ Composição Granulométrtica da Terra Fina $\left(\mathrm{g} \mathrm{kg}^{-1}\right)$} & \multicolumn{6}{|c|}{ Complexo Sortivo $\left(\mathrm{cmol}_{\mathrm{c}} \mathrm{kg}\right)$} \\
\hline & & Areia Grossa & Areia Fina & Silte & Argila & $\mathrm{Ca}^{2+}$ & $\mathrm{Mg}^{2+}$ & $\mathrm{K}^{+}$ & $\mathrm{Na}^{+}$ & $\mathrm{Al}^{3+}$ & $\mathrm{H}^{+}$ \\
\hline Ap & $0-20$ & 460 & 80 & 170 & 290 & 2,5 & 2,2 & 0,26 & 0,04 & 0 & 3,3 \\
\hline
\end{tabular}


onde as amostras foram colocadas em becker de $1 \mathrm{~L}$, sendo possível a determinação do volume escoado, em seguida, os mesmos foram colocados sobre manta elétrica possibilitando a evaporação parcial da água. Visando à completa evaporação da água e a determinação da massa de solo presente em cada amostra, as mesmas foram colocadas em estufa, numa temperatura de $105^{\circ} \mathrm{C}$, pelo período de $24 \mathrm{~h}$, a partir do qual, a massa de solo presente em cada amostra foi determinada em balança de precisão.

Para a análise estatística foi utilizado o programa SAEG (Sistema de Análises Estatística e Genética) a fim de se obter equações de regressão que melhor representassem a variação das perdas de solo e água, em função do tempo de aplicação da chuva; posteriormente, foi utilizada a metodologia de Steel \& Torrie (1980) (Eq. 1) para verificar a homogeneidade dos coeficientes de regressão obtidos.

$$
\mathrm{t}=\frac{\mathrm{b}_{1}-\mathrm{b}_{2}}{\sqrt{\left(\frac{\mathrm{SQRreg}{ }_{1}+\mathrm{SQRreg}_{2}}{\mathrm{n}_{1}+\mathrm{n}_{2}-4}\right)\left(\frac{1}{\mathrm{SQx}_{1}}+\frac{1}{\mathrm{SQx}_{2}}\right)}}
$$

em que:

t - parâmetro calculado

$b_{i} \quad$ - coeficiente angular do modelo de regressão

$\mathrm{SQRreg}_{\mathrm{i}}$ - soma de quadrado do resíduo da regressão

$\mathrm{n}_{\mathrm{i}} \quad$ - número de dados utilizados na análise

$\mathrm{SQX}_{\mathrm{i}}$ - soma de quadrado do resíduo

\section{RESULTADOS E DISCUSSÃO}

A Tabela 2 apresenta os valores médios de massa de solo e volume de água escoado no final da aplicação das chuvas simuladas com diferentes energias cinéticas. Nota-se que, à medida que a energia cinética aumentou, maiores valores de perda de solo foram verificados e, em razão do aumento do volume precipitado, maiores valores de escoamento superficial foram coletados.

Tabela 2. Perdas de solo $\left(\mathrm{g} \mathrm{m}^{-2}\right)$ e água $\left(\mathrm{m}^{3} \mathrm{~m}^{-2}\right)$ em função dos valores de energia cinética das chuvas simuladas

\begin{tabular}{|c|c|c|c|c|c|}
\hline \multirow[b]{2}{*}{ Tempo } & \multicolumn{5}{|c|}{ Energia Cinética da Chuva Simulada $\left(\mathrm{J} \mathrm{m}^{-2}\right)$} \\
\hline & 138 & 184 & 229 & 275 & 321 \\
\hline \multicolumn{6}{|c|}{ A. Perda do solo $\left(\mathrm{g} \mathrm{m}^{-2}\right)$} \\
\hline 5 & 0,85 & 0,67 & 0,97 & 1,07 & 1,30 \\
\hline 10 & 1,10 & 1,54 & 2,26 & 2,46 & 3,29 \\
\hline 15 & 1,61 & 2,65 & 3,27 & 4,22 & 6,28 \\
\hline 20 & 1,80 & 3,81 & 4,78 & 6,13 & 9,16 \\
\hline 25 & 2,09 & 5,13 & 6,76 & 8,50 & 17,36 \\
\hline 30 & 2,50 & 6,49 & 8,28 & 11,29 & 22,73 \\
\hline 35 & 2,86 & 7,90 & 9,86 & 13,39 & 27,73 \\
\hline \multicolumn{6}{|c|}{ B. Perda de água $\left(\mathrm{m}^{3} \mathrm{~m}^{-2}\right)$} \\
\hline 5 & 0,00022 & 0,00037 & 0,00054 & 0,00055 & 0,00079 \\
\hline 10 & 0,00050 & 0,00093 & 0,00135 & 0,00163 & 0,00212 \\
\hline 15 & 0,00080 & 0,00163 & 0,00237 & 0,00287 & 0,00386 \\
\hline 20 & 0,00103 & 0,00241 & 0,00359 & 0,00433 & 0,00580 \\
\hline 25 & 0,00135 & 0,00338 & 0,00490 & 0,00577 & 0,00822 \\
\hline 30 & 0,00175 & 0,00455 & 0,00631 & 0,00710 & 0,01110 \\
\hline 35 & 0,00221 & 0,00584 & 0,00783 & 0,00933 & 0,01418 \\
\hline
\end{tabular}

Com os dados das Tabela 2A e B, foram ajustadas equações de regressão, tendo como variáveis dependentes a massa de solo e o volume de água escoado e como variável independente, o tempo de duração da chuva simulada. Nas Figuras 1A e B estão apresentadas as equações geradas com os respectivos coeficientes de determinação e as curvas ajustadas a partir dessas equações, para as perdas de solo e água, respectivamente. Verifica-se que, para os menores valores de energia cinética, o modelo selecionado para o ajuste da massa de solo foi o linear, porém a partir de $275 \mathrm{~J} \mathrm{~m}^{-2}$, o comportamento potencial foi verificado. Essa mudança se baseia no fato de que as partículas de solo arrastadas por baixas taxas de escoamento, são parcialmente depositadas dentro da própria parcela experimental, não sendo computadas como perda efetiva de solo. Para o volume de água escoado, o melhor ajuste observado foi o potencial, para todos os valores de energia cinética observados.

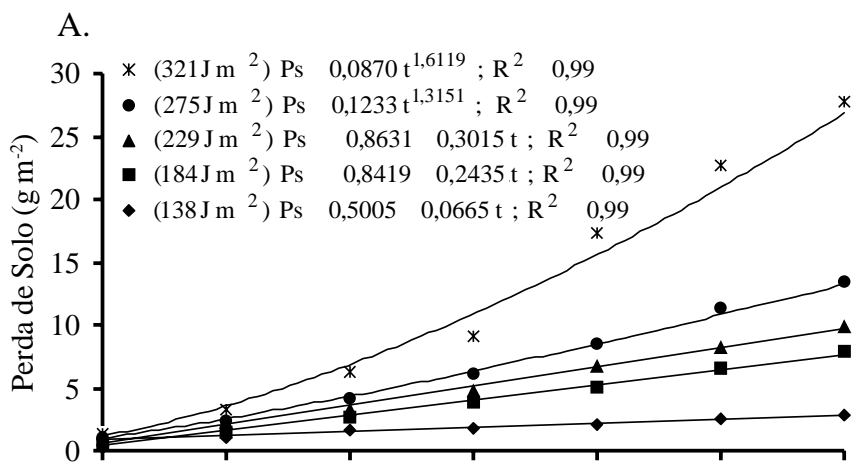

B.

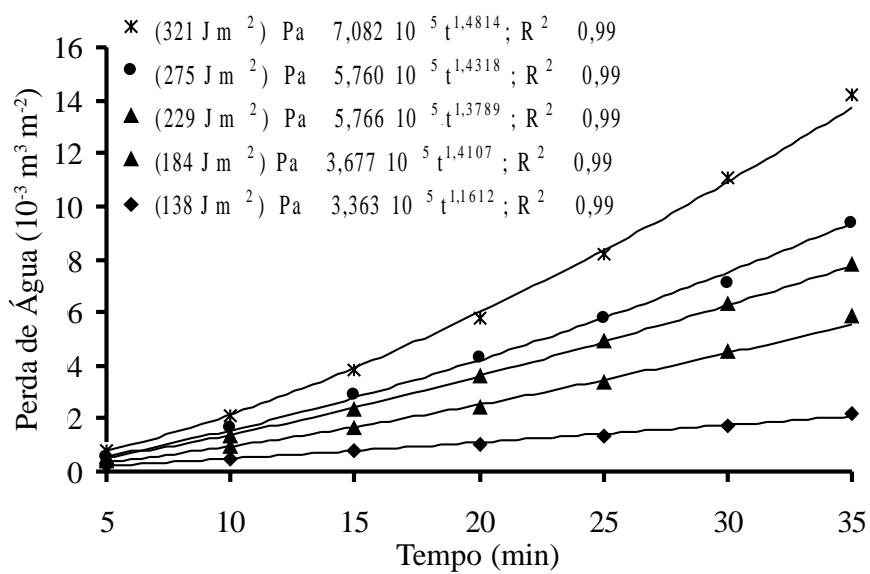

Figura 1. Perda acumulada de solo (A) e água (B) em função do tempo de aplicação da chuva simulada

Vale a pena ressaltar que, da mesma forma como apresentado por Amorim et al. (2001), com um aumento dos valores de energia cinética, foi observado um aumento da vazão de escoamento superficial proporcionado pelo aumento da intensidade de precipitação. Este fato pode ter ocasionado uma concentração do escoamento, com a formação de caminhos preferenciais de escoamento de água aumentando, a partir de certo tempo de aplicação, a sua capacidade de transporte.

A fim de se verificar a qualidade dos modelos ajustados, valores de perda de solo e água obtidos pelas equações ajustadas foram comparados com aqueles medidos no campo. 
A Tabela 3 apresenta os resultados da comparação, considerando-se precipitações simuladas com 35 min de duração.

Tabela 3. Comparação entre os valores simulados e medidos de perdas de solo e água, para precipitações simuladas com 35 min de duração

\begin{tabular}{crrr}
\hline \multirow{2}{*}{ Energia Cinética $\left(\mathrm{J} \mathrm{m}^{-2}\right)$} & \multicolumn{2}{c}{ Valores } & \multirow{2}{*}{ Variação } \\
\cline { 2 - 3 } & Simulados & Medidos & \\
\hline A. Massa de solo $\left(\mathrm{g} \mathrm{m}^{-2}\right)$ & 2,83 & 2,86 & $-0,011$ \\
138 & 7,68 & 7,90 & $-0,028$ \\
184 & 9,69 & 9,86 & $-0,018$ \\
229 & 13,23 & 13,39 & $-0,012$ \\
275 & 26,82 & 27,73 & $-0,034$ \\
321 & & & \\
B. Volume escoado $\left(\mathrm{m}^{3} \mathrm{~m}^{-2}\right)$ & 0,00209 & 0,00221 & $-0,057$ \\
138 & 0,00554 & 0,00584 & $-0,054$ \\
184 & 0,00776 & 0,00783 & $-0,009$ \\
229 & 0,00936 & 0,00933 & 0,003 \\
275 & 0,01370 & 0,01418 & $-0,035$ \\
321 & & & \\
\hline
\end{tabular}

Tendo em vista os altos coeficientes de determinação obtidos nas análises de regressão, observam-se pequenas variações entre os valores simulados pelas equações e os valores medidos. Com exceção do volume escoado a partir da chuva simulada com energia de $275 \mathrm{~J} \mathrm{~m}^{-2}$, as equações ajustadas subestimaram os valores medidos, atingindo a variação de 3,4 e $5,7 \%$, para as perdas de solo e água, respectivamente.

Trabalhando em uma área com o mesmo tipo de solo e cultivada sob plantio convencional (aração seguida de gradagem), Varella (1999) utilizando o mesmo simulador de chuvas, encontrou uma perda de solo de $17 \mathrm{~g} \mathrm{~m}^{-2}$, após $35 \mathrm{~min}$ de aplicação de uma chuva simulada com uma energia cinética de $288 \mathrm{~J} \mathrm{~m}^{-2}$. Nessas mesmas condições, o volume de água escoado após $35 \mathrm{~min}$ foi de $0,01349 \mathrm{~m}^{3} \mathrm{~m}^{-2}$. Em razão do manejo com que a área experimental do SIPA vem sendo conduzida, verifica-se que a presença de restos culturais na superfície associada à pequena movimentação mecânica do solo minimiza o impacto das gotas, diminuindo a desagregação do solo e, conseqüentemente, a concentração de sedimentos na enxurrada. Resultados semelhantes foram encontrados por Leite et al. (2002). Trabalhando em um Nitossolo Háplico Alumínico (Terra Bruna Estruturada) de textura argilosa, foram encontradas perdas que variaram de $0,00023 \mathrm{~g} \mathrm{~m}^{-2}$, em solo com semeadura direta em campo dessecado, a $0,0654 \mathrm{~g} \mathrm{~m}^{-2}$, para a condição de solo sem cobertura.

As Tabelas 4 e 5 apresentam os resultados dos testes de homogeneidade dos coeficientes de regressão dos modelos ajustados para a perda de solo e água, respectivamente. Apesar das diferenças visuais observadas entre os modelos ajustados (Figs. 1A e B), pode-se notar que nem todos diferem estatisticamente entre si.

Para perda de solo (Tabela 4) o coeficiente angular do modelo ajustado para o tratamento com energia cinética de $138 \mathrm{~J} \mathrm{~m}^{-2}$ não difere estatisticamente dos coeficientes ajustados para a energia cinética de 184 e $229 \mathrm{~J} \mathrm{~m}^{-2}$, a nível de $5 \%$ de probabilidade. $\mathrm{O}$ mesmo aconteceu entre os tratamentos com energias cinéticas de 184 e $229 \mathrm{~J} \mathrm{~m}^{-2}$. Para os demais contrastes, este efeito não foi observado, resultando em modelos estatisticamente diferentes, em nível de $1 \%$ de probabilidade.
Tabela 4. Estudo da homogeneidade dos coeficientes angulares de regressão dos modelos estimados para a perda de solo, entre os tratamentos considerados

\begin{tabular}{|c|c|c|c|c|}
\hline Contraste & $b_{1}-b_{2}$ & SQRreg ${ }^{1}$ & $\mathrm{t}_{\mathrm{cal}}^{2}$ & Teste \\
\hline $\mathrm{T}=138 \operatorname{com} \mathrm{T}=184$ & $-0,177$ & 0,09444 & $-1,87$ & $\mathrm{~ns}$ \\
\hline $\mathrm{T}=138 \operatorname{com} \mathrm{T}=229$ & $-0,235$ & 0,11530 & $-2,03$ & ns \\
\hline $\mathrm{T}=138 \mathrm{com} \mathrm{T}=275$ & $-0,2815$ & 0,01718 & $-16,38$ & $* *$ \\
\hline $\mathrm{T}=138 \operatorname{com} \mathrm{T}=321$ & $-0,5819$ & 0,01968 & $-29,57$ & ** \\
\hline $\mathrm{T}=184 \operatorname{com} \mathrm{T}=229$ & 0,058 & 0,14500 & 0,40 & ns \\
\hline $\mathrm{T}=184 \operatorname{com} \mathrm{T}=275$ & 0,1885 & 0,02052 & 9,19 & $* *$ \\
\hline $\mathrm{T}=184 \operatorname{com} \mathrm{T}=321$ & $-0,1119$ & 0,02265 & $-4,94$ & $* *$ \\
\hline $\mathrm{T}=229 \operatorname{com} \mathrm{T}=275$ & $-0,1615$ & 0,01798 & $-8,98$ & $* *$ \\
\hline $\mathrm{T}=229 \operatorname{com} \mathrm{T}=321$ & $-0,4619$ & 0,02040 & $-22,64$ & $* *$ \\
\hline $\mathrm{T}=275 \operatorname{com} \mathrm{T}=321$ & $-0,3004$ & 0,02143 & $-14,02$ & $* *$ \\
\hline
\end{tabular}

Tabela 5. Estudo da homogeneidade dos coeficientes angulares de regressão dos modelos estimados para a perda de água, entre os tratamentos considerados

\begin{tabular}{crrrc}
\hline Contraste & $\mathrm{b}_{1}-\mathrm{b}_{2}$ & $\mathrm{SQRreg}^{1}$ & $\mathrm{t}_{\text {cal }}{ }^{2}$ & Teste \\
\hline $\mathrm{T}=138 \operatorname{com} \mathrm{T}=184$ & $-0,24952$ & 0,03570 & $-6,99$ & $* *$ \\
$\mathrm{~T}=138 \operatorname{com} \mathrm{T}=229$ & $-0,21767$ & 0,02790 & $-7,80$ & $* *$ \\
$\mathrm{~T}=138 \operatorname{com} \mathrm{T}=275$ & $-0,27059$ & 0,36800 & $-7,35$ & $* *$ \\
$\mathrm{~T}=138 \operatorname{com} \mathrm{T}=321$ & $-0,32018$ & 0,03051 & $-10,49$ & $* *$ \\
$\mathrm{~T}=184 \operatorname{com} \mathrm{T}=229$ & 0,03185 & 0,02614 & 1,22 & $\mathrm{~ns}$ \\
$\mathrm{~T}=184 \operatorname{com} \mathrm{T}=275$ & $-0,02107$ & 0,03680 & $-0,72$ & $\mathrm{~ns}$ \\
$\mathrm{~T}=184 \operatorname{com} \mathrm{T}=321$ & $-0,07066$ & 0,02920 & $-2,53$ & $*$ \\
$\mathrm{~T}=229 \operatorname{com} \mathrm{T}=275$ & $-0,05292$ & 0,02790 & $-1,90$ & $\mathrm{~ns}$ \\
$\mathrm{~T}=229 \operatorname{com} \mathrm{T}=321$ & $-0,10251$ & 0,01893 & $-5,41$ & $* *$ \\
$\mathrm{~T}=275 \operatorname{com} \mathrm{T}=321$ & $-0,04959$ & 0,03060 & $-1,62$ & $\mathrm{~ns}$
\end{tabular}

${ }^{1}$ Soma de quadrado do resíduos da regressão conforme Eq. 1

${ }^{2} \mathrm{t}_{(1 \%, 10)}=3,169 ; \mathrm{t}_{(5 \%, 10)}=2,228$

** - Significativo a $1 \%$ de probabilidade, pelo teste $\mathrm{t}$

* - Significativo a $5 \%$ de probabilidade, pelo teste $\mathrm{t}$

ns - Não significativo, pelo teste t

Analisando-se a Tabela 5, percebem-se resultados distintos com relação às perdas de solo, uma vez que os tratamentos com maiores intensidades de precipitação simuladas são aqueles cujos coeficientes angulares ajustados não diferiram, em nível de 5\% de probabilidade.

Apesar da análise estatística apresentar resultados não significativos entre alguns tratamentos percebe-se, no entanto, que, sob o ponto de vista físico, há uma variação expressiva nos valores de perda de solo e água, obtidos para as diferentes chuvas simuladas (Tabela 3). Além disso, tomando ainda como base os valores apresentados, observa-se o efeito da energia cinética nas perdas de solo e água se observa um aumento de 9,7 vezes na perda de solo e de 6,4 vezes na perda de água, quando o valor da energia cinética foi aumentado de 138 para $321 \mathrm{~J} \mathrm{~m}^{-2}$. Young \& Wiersma (1973) verificaram que, para uma redução de $89,0 \%$ da energia de impacto das gotas, ocorreu decréscimo de perda de solo superior a 90,0\%. Esse mesmo comportamento foi também obtido por Amorim et al. (2001) que, estudando a influência da energia cinética em chuvas simuladas no processo de erosão entre sulcos, verificaram aumento de 44,7 vezes na perda de solo, quando a energia cinética aumentou de 495 para $1959 \mathrm{~J} \mathrm{~m}^{-2}$, para chuvas simuladas com 58 min de duração e uma declividade da superfície do 
solo de $10 \%$. Na condução desse experimento, os autores trabalharam em um canal de solo no qual foi colocada uma camada de solo de aproximadamente $150 \mathrm{~mm}$ de espessura, ficando o mesmo exposto à precipitação simulada.

\section{CONCLUSÕES}

1. A perda acumulada de solo apresentou comportamento linear ao longo dos testes com chuvas simuladas, para valores de energia cinética inferiores a $275 \mathrm{~J} \mathrm{~m}^{-2}$.

2. Para todos os valores de energia cinética utilizados, a perda acumulada de água apresentou comportamento potencial, indicando um crescimento gradativo da mesma, ao longo dos testes.

3. As perdas aumentaram com o incremento da energia cinética da precipitação, atingindo 9,7 vezes na perda de solo e 6,4 vezes na perda de água, quando o valor da energia cinética das chuvas foi aumentado de 138 para $321 \mathrm{~J} \mathrm{~m}^{-2}$, respectivamente.

4. A utilização de equipamentos, como o simulador de chuvas, constitui importante ferramenta para a quantificação e o entendimento do processo erosivo em parcelas experimentais.

\section{LITERATURA CITADA}

Alves Sobrinho, T.; Carvalho, D.F.; Aquino, R.M.; Montebeller, C.A. Programa computacional para a definição de parâmetros hidráulicos utilizados na determinação da energia cinética da chuva simulada em infiltrômetros de aspersão. Engenharia Rural, Piracicaba, v.12, n.1, p.28-35, 2001.

Amorim, R.S.S. Desprendimento e arraste de partículas de solo decorrentes de chuvas simuladas. Viçosa: UFV, 1999. 75p. Dissertação Mestrado

Amorim, R.S.S; Silva, D.D.; Pruski, F.F.; Matos, A.T. Influência da declividade do solo e da energia cinética de chuvas simuladas no processo de erosão entre sulcos. Revista Brasileira de Engenharia Agrícola e Ambiental, Campina Grande, v.5, n.1, p.124-130, 2001.
Bahia, V.G.; Curi,N.; Carmo,D.; Marques, J.J.G.M.M. Fundamentos de erosão do solo. Informe Agropecuário. Belo Horizonte, v.16, xa n.176, p.25-31, 1992.

Bertoni, J; Lombardi Neto, F. Conservação do solo. Piracicaba: Editora Livroceres, 1985. 392p.

Chaves, H.M.L; Orlowski, E.; Roloff, G. Previsão da infiltração sob condições dinâmicas de selamento superficial. Revista Brasileira de Ciência do Solo, Campinas, v.17, n.2, p.141-147, 1993.

Kinnell, P.I.A.; Cummings, D. Soil slope gradient interactions in erosion by rain-impacted flow. Transactions of the ASAE, St. Joseph, v.36, n-2, p.381-387, 1993.

Leite, D.; Bertol, I.; Zaporalli, AS.; Carrafa, R. Erosão hídrica sob chuva simulada no milho. In: Reunião Brasileira de Manejo e Conservação do Solo e da Água, 14, 2002, Cuiabá. Anais...Cuiabá: Sociedade Brasileira de Ciência do Solo, 2002. CD-Rom

Montebeller, C.A.; Carvalho, D.F.; Alves Sobrinho, T.; Nunes, A.C.S.; Rubio, E. Avaliação hidráulica de um simulador de chuvas pendular. Revista Brasileira de Engenharia Agrícola e Ambiental, Campina Grande, v.5, n.1, p.1-5, 2001.

Morin, J; van Winkel, J. The effect of raindrop impact and sheet erosion on infiltration rate and crust formation. Soil Science Society of America Journal, Madison, v.60, p.1223-1227, 1996.

Pruski, F.F. Conservação de água e solos. Brasília, DF: ABEAS; Viçosa, MG: UFV/DEA, 1996. 88p. Curso de Gestão de Recursos Hídricos para o Desenvolvimento Sustentados de Projetos Hidroagrícolas. Módulo, 7

Reichert, J. M; Veiga, M; Cabeda, M. S. V. Selamento superficial e infiltração de água em solos do Rio Grande do Sul. Revista Brasileira de Ciência do Solo, Campinas, v.16, n.3, p.289-298, 1992.

Steel, R.G.D.; Torrie, J.H. Principles and procedures of statistics. New York: Mc-Graw-Hill Book Company, 1980. 633p.

Varella, C. A. A. Efeito dos sistemas de cultivo nas perdas de água e solo. Seropédica, RJ: UFRRJ, 1999, 93p. Dissertação Mestrado

Young, R.A. Wiersma, J. The role of rainfall impact in soil detachment and transport. Water Resources Research, Washington, v.9, n.6, p.1629-1636, 1973. 